\title{
APPLYING THE RADIO RANGE TO THE AIRWAYS
}

\author{
By F. G. Kear and W. E. Jackson
}

\section{ABSTRACT}

In pursuance of the program of radio aids to flight undertaken by the Department of Commerce, design and installation work has progressed under the direction of the chief engineer, airways division, in the application of the radio range (directive radiobeacon) along the airways. To date, only the aural type has been put into routine daily operation, and it is the only type of beacon considered in this paper which discusses methods of adjusting the space pattern of the beacon system in order that the courses may align with the fixed airways. These beacons also need to be readily distinguished from one another and so designed that a minimum of interference is met.

By using a vertical wire antenna in addition to the loop antennas, and varying the relative power in the two loop antennas, it was found possible to secure practically any array of courses desired. The radio ranges at Hadley Field, N. J., and Bellefonte, Pa., were employed in the experimental work with excellent results. The field intensity measurements made gave space patterns which checked very well with the theoretical patterns for such antenna systems.

Careful spacing of the radio range within the frequency band, as well as distinctive coding of each beacon, solved the problem of interference.

In selecting the proper coding for the beacons a study of the physiological effects of various sound groups was made, and the final coding chosen was such as to give a signal of equal time duration on each side of the course. The signals still interlock to give the customary long dash when on the course.

The technique of applying those modifications was developed to the point where the adjustments could be made by the installation crew in the field without the aid of an engineer.

\section{CONTENTS}

Page

I. Introduction

II. Interference between radio ranges

III. Alignment of courses with airways

IV. Application of theory 378

\section{INTRODUCTION}

The aural type of the radio range ${ }^{1}$ was installed by the airways division at Hadley Field, N. J., Bellefonte, Pa., and Cleveland, Ohio, in 1927 and 1928. After the first tests, it became necessary to adjust the beacon system in order to make the courses provided coincide with the fixed airways. The original aural type radio range established an equisignal zone of about $3^{\circ}$ width extending in a straight line through the beacon approximately 100 miles either way. Another equisignai zone, at right angles thereto, approximately $12^{\circ}$ in width, was also present, but on account of its excessive width was not usable. An airway extending 200 miles in a straight line with a field at its center is a situation seldom encountered. The use of

1 F. H. Engel and F. W. Dunmore, A Directive Type of Radiobeacon and its Application to Navigation, B.S. S. Papers No. 480; January, 1924. Murphy and Wolfe, Stationary and Rotating Equisignal Beacons, J. S. A. E., 19, 209; September, 1926. Dellinger and Pratt, Development of Radio Aids to Air Navigation, Proc. I. R. E., 16, pp. 890-920; July, 1928. 
lower power beacons at more frequent intervals, and between fields, might solve this difficulty, but would raise problems of interference and economy. In the actual use of the beacons along the airways the following problems had to be solved:

(a) Interference had to be kept to a minimum in order to prevent confusion.

(b) The course outlined had to be easily recognizable so that a slight deviation might be noticed.

(c) Radio ranges had to be so designed that they could be located at bends in the airway route; that is, the course given by the beacon must not be a straight line through the beacon, but an obtuse angle of controllable magnitude. Furthermore, both legs of the angle must be capable of simultaneous operation in order to accommodate increasing air traffic.

(d) Finally, it was necessary to service more than one course from a single radio range, a further development of the previous idea. Terminals in larger cities have many air routes entering, and each of these routes should be outlined by a beacon course. Since it would not be economically possible to install a separate beacon for each route the system had to be modified to accommodate multiple routes.

These conditions have all been satisfied on the radio range system as now in use on the airways, if the number of courses to be served does not exceed four. The purpose of this paper is to give a detailed account of the means employed to meet these conditions.

\section{INTERFERENCE BETWEEN RADIO RANGES}

Two methods have been successfully adopted to differentiate between radio ranges. The first method was to allocate different frequencies in the band from 285 to $350 \mathrm{kc}$. to each radio range. Particular care was taken to separate adjacent beacon frequencies by at least $12 \mathrm{kc}$. When utilizing the ordinary aircraft receiver using two stages of shield grid tube, radio-frequency amplification, detector and two stages audio with a 6 -foot rod antenna, it was possible to tune in either a beacon 100 miles ahead or 100 miles behind, when both were operating simultaneously on frequencies separated by 8 lic. The average power output from a beacon installation is $2 \mathrm{kw}$.

The second method used to eliminate possible confusion between radio ranges was to establish a system of distinctive code signals. The signals of necessity had to be such that they interlocked and gave the resulting long dash when on the course. The first group of signals adopted were $(-$ and.-$) ;(-.-$ and..$) ;(-\ldots-$ and $\ldots .$.$) ;$ and ( $-\ldots$ and ...); two characteristic signals being required for each interlocking radio range. Results showed that radio ranges using the $\ldots$ and . - characteristics gave very distinctive courses. However, when the characters _ . - and .. were used as interlocking signals, a peculiar physiological effect was observed. Tests indicated that the course was apparen tly bent slightly in an S shape. In reality, field strength measurements proved the course to be straight. When close to the range and on the course as indicated by field-intensity measurement, the character - - would apparently predominate, causing the pilot to bear toward the .. side of the course. When at approximately 100 miles from the range, tests indicated that the .. character would predominate while on the course, thus causing the 


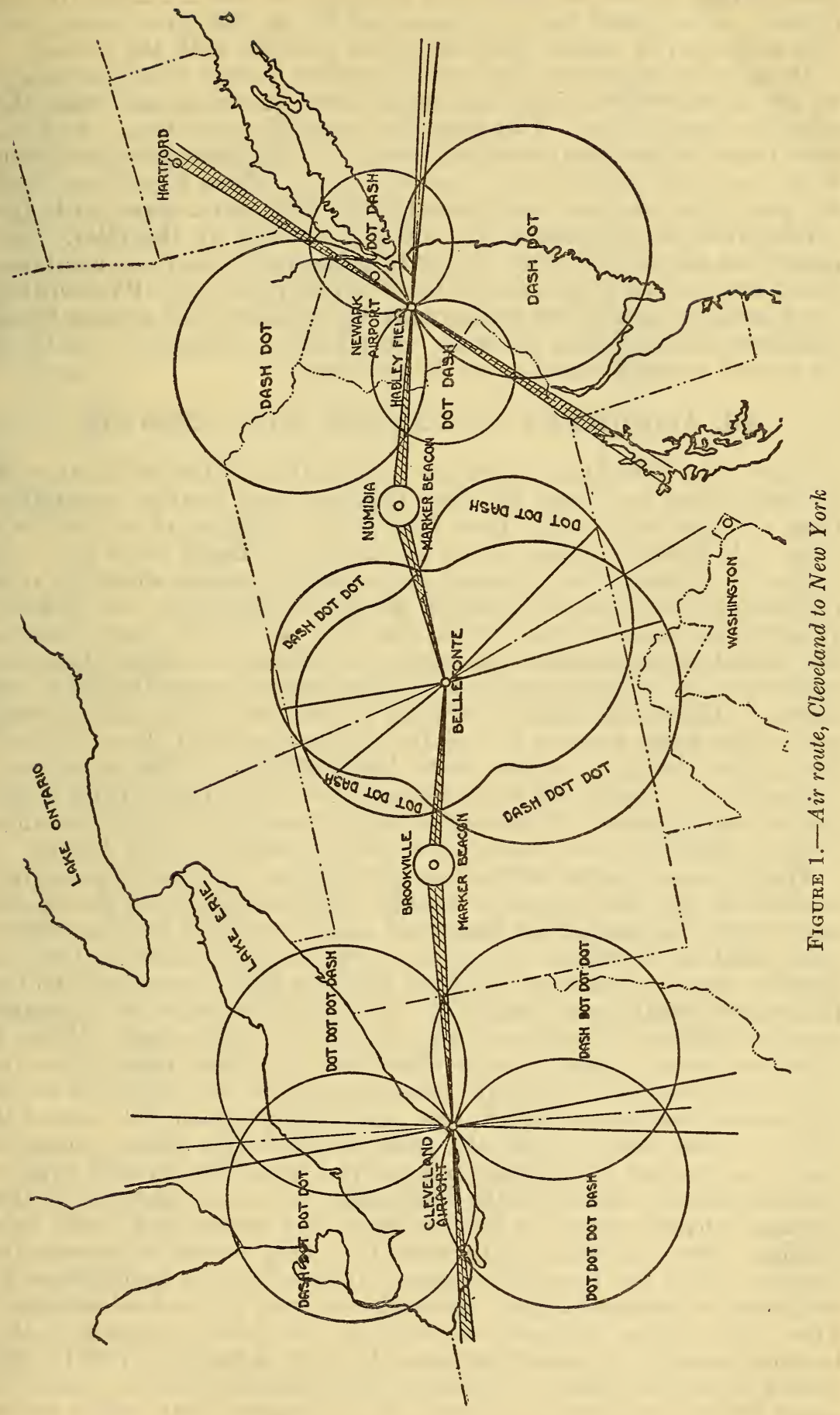


pilot to compensate for this effect by flying on the - . - side of the course. As a result the pilot does not fly on the true course, but flies on a curved course which does not coincide with the airway.

In order to eliminate this confusing effect it was found necessary to use characteristics that had equal time durations and were the inverse of each other. This plan was adopted since the - . and .were found to give such excellent results. The characteristics chosen were $(\ldots$ and $\ldots-) ;(\ldots .$. and $\ldots .$.$) . These have been used$ for some time and have eliminated difficulties encountered with dissymmetrical arrangements, but are not favored by the pilot. Recently the airways division changed signals to - . and . - combinations, transmitted in groups of 2 to 12 signals per cycle. By counting the number of signals the station may be identified, this system being similar to a clock tolling the hour. In addition, beacons are identified by station announcement every 15 minutes.

\section{ALIGNMENT OF COURSES WITH AIRWAYS}

It has long been appreciated that a limitation of the radio range in its early form has been the fact that the four courses emanating from a single radio range have been at fixed angles of $90^{\circ}$ to each other. It is obvious that such an arrangement could serve only one course at a time when situated at an airport except where by rare coincidence two courses extended at $90^{\circ}$ or $180^{\circ}$ from an airport. In order to make at least two of the four available courses useful the radiobeacon installations could be situated halfway between points on the straight airways at distances of approximately 200 miles. This would make it possible to use two of the four courses and at the same time to reduce the angular width of the equisignal zone since the two courses were displaced $180^{\circ}$. This is accomplished by increasing the angle between the two primary coils of the goniometer to about $120^{\circ}$ instead of $90^{\circ}$. (See fig. 4.) Some installations of this type were made along the Transcontinental Airway.

Placing radio ranges in this manner has one particularly annoying feature in that the course is usually broadest where it should be narrowest. To make this statement clear, it should first be understood that an equisignal course is a true angular function; that is, directly over the center of the loop antenna the courses have an infinitesimal width, and gradually the width increases to approximately 6 miles at 100 miles distance from the radio range. When a pilot flies from a point at a distance from the radio range it is evident that his course gradually becomes sharper and narrower as he approaches the beacon installation, and if he keeps on his course it will take him directly over the radio tower. This feature alone is one of paramount importance and can not be neglected, since experience has shown that when the radio range is located adjacent to the terminal airport pilots are able to locate the airport and make safe landings when conditions are such that they would otherwise be unable to find the terminal airport. Hence, when a radiobeacon is located at a terminal airport there are two very distinct advantages: First, the course becomes narrower as the pilot approached the beacon; second, the beacon informs the pilot definitely when he has passed over the tower. Therefore, it practically fixes a point in space directly over the antennas. It is apparent that such a point 


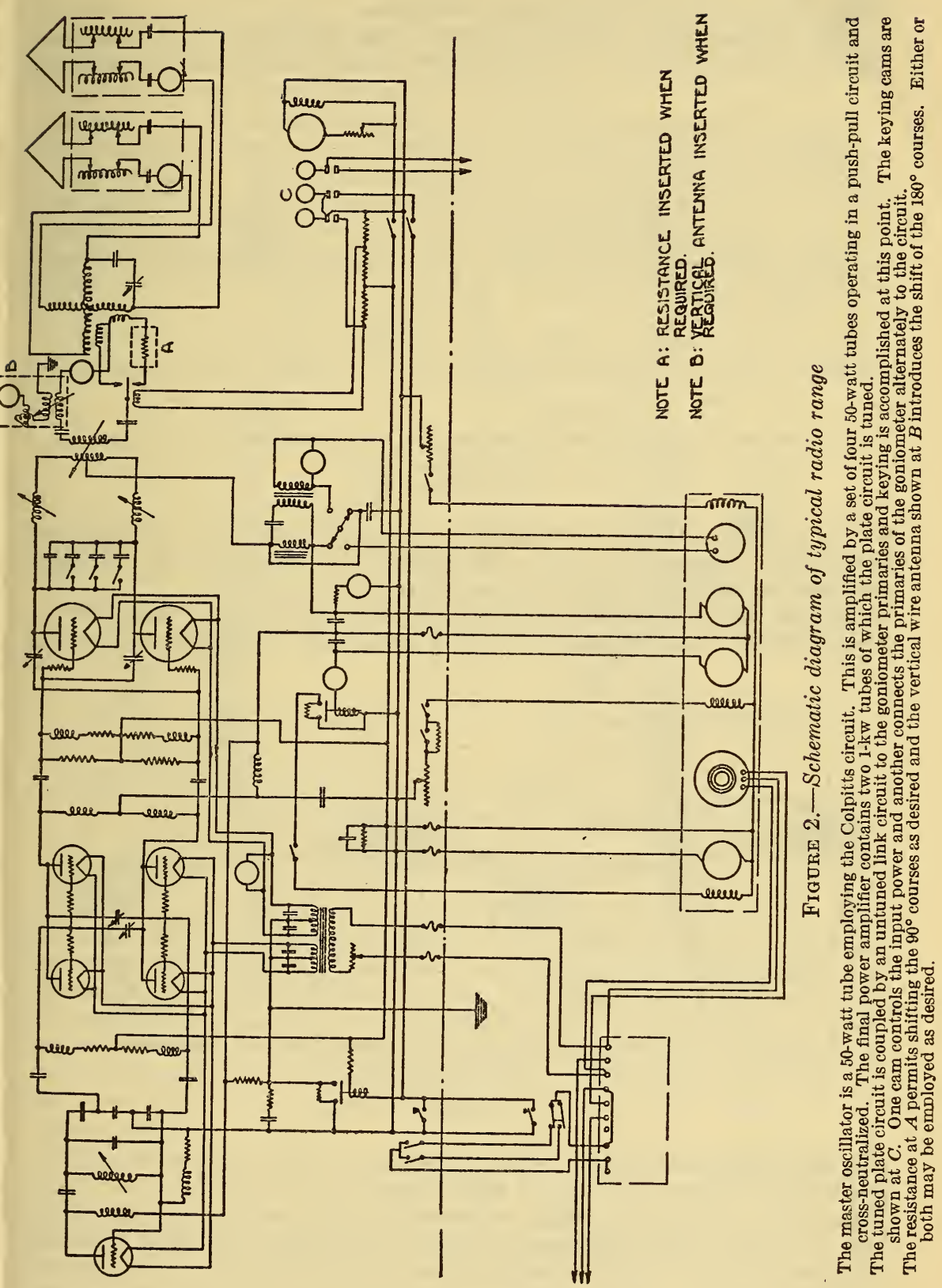

$92380^{\circ}-30-4$ 
in space would be of unquestionable value if located adjacent to a terminal airport instead of halfway between two airports. It was with these particular features in mind that the problem of fitting radio ranges to the airways was attacked.

Two fundamental schemes have been developed which permit the use of two fixed courses displaced at any angle varying between

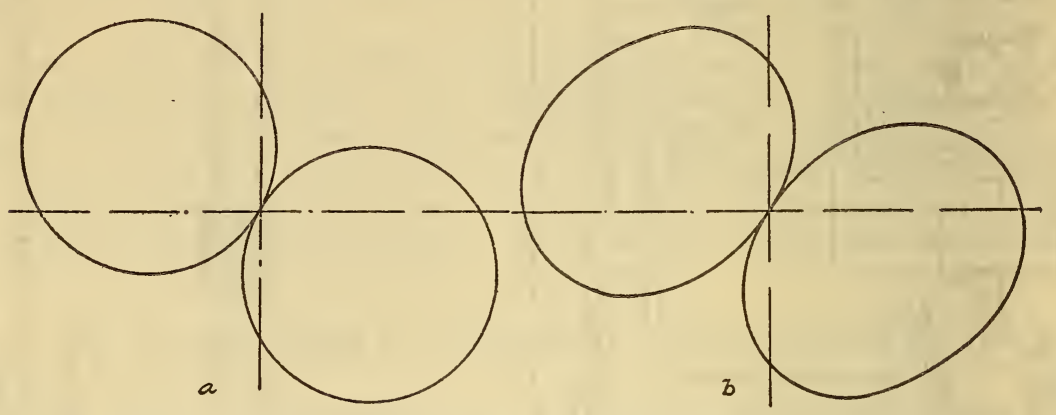

FIGURE 3.-Field intensity patiern of a single phantom loop antenna

(a) Theoretical.

(b) Observed.

$45^{\circ}$ and $180^{\circ}$. Courses emanating at angles less than $45^{\circ}$ can not practically be serviced with one installation, because an excessive decrease in power results from such an attempt.

Method used for courses emanating at angles between $45^{\circ}$ and $135^{\circ}$.The method used to serve courses emanating from an airport at
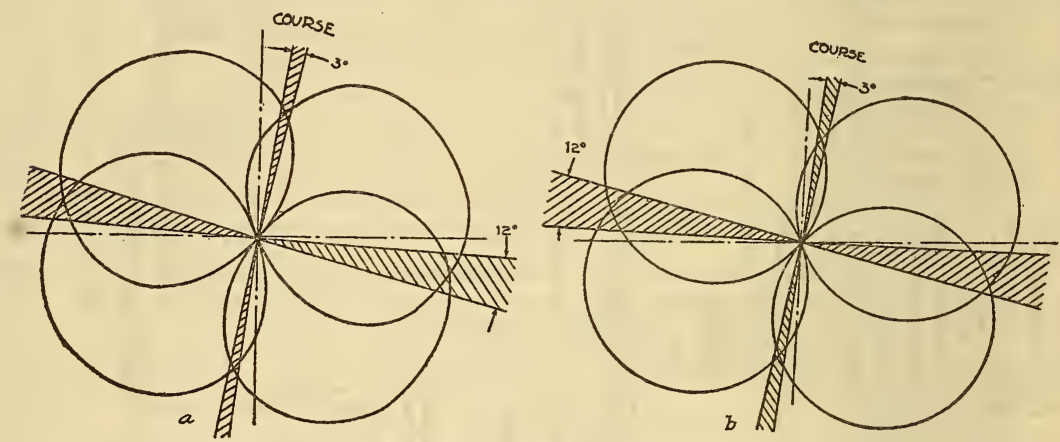

FIGURE 4.-Field intensity pattern of typical radio range. Goniometer primaries set at $120^{\circ}$

(a) Theoretical.

(b) Observed.

angles varying between $45^{\circ}$ and $135^{\circ}$ is as follows: A noninductive resistance is inserted in the link circuit in series with one of the goniometer primaries. This reduces the power in one phantom loop antenna ${ }^{2}$ while the power in the other phantom loop antenna is left unaffected. By varying the ratio of power in the two phantom

2 A phantom loop antenna is the resultant figure-of-eight space pattern occurring when both loop an tennas are coupled to one goniometer primary. One phanton loop antenna exists in space for each goniometer primary. The angular relation of the phantom loop antennas is the same as that of the goniometer primaries. For a further treatment of this subject see Murphy and Wolfe, Stationary and Rotating Equisignal Beacons, J. Soc. of Automotive Engs., 19, p. 209, September, 1926. 
loop antennas by means of resistance the courses may be made to shift from their normal $90^{\circ}$ displacement to any angle between $45^{\circ}$ and $135^{\circ}$. (See fig. 5.) It should be carefully noted that this variation depends upon several variables, including loop antenna. resistance, mutual inductance between primary and secondary of goniometer, and power factor of link circuit. These should be taken care of jointly in each particular installation.

Method used for courses emanating at angles between $135^{\circ}$ and $180^{\circ}$.The normal polar pattern of crossed loop antennas is a pair of figuresof-eight at $90^{\circ}$. This gives four equisignal zones which are referred to as "courses" in beacon terminology. (See fig. 6.) In order to increase the sharpness of the course the goniometer primaries are frequently set at $120^{\circ}$ instead of $90^{\circ}$. This gives four courses, two of which are about $3^{\circ}$ wide, while the remaining two are $12^{\circ}$ wide. (See fig. 4.)

If a vertical wire is now permitted to radiate in time phase with the radiation from the phantom loop antenna the field intensity pattern

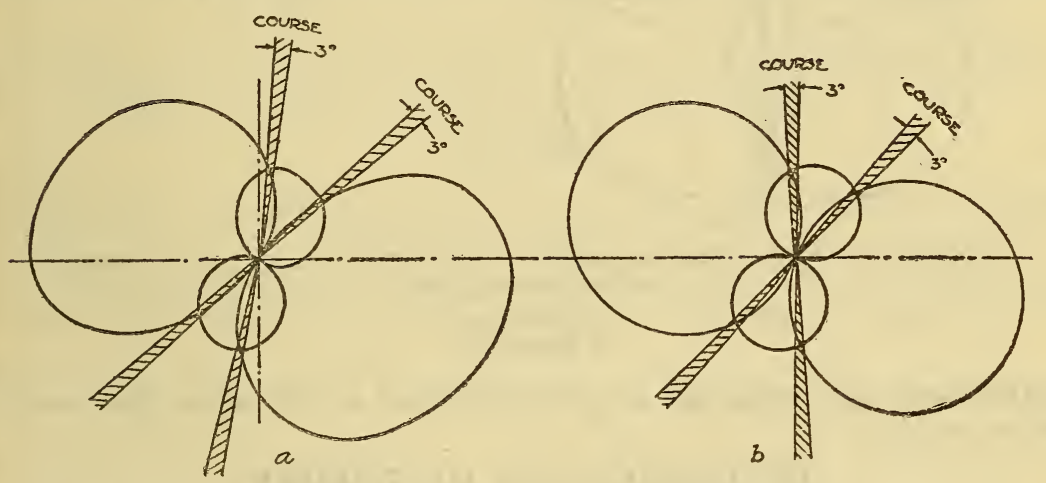

FIgURE 5.-Field intensity pattern of radio range with resistance inserted in series with one goniometer primary. Primaries set at $90^{\circ}$

(a) Observed.

(b) Theoretical.

will be distorted as shown in Figures 7 and 8 . It will be noted that two lobes diminish and two increase. This changes the relative position of the equisignal zones so that the course approaching the beacon is no longer $180^{\circ}$ from the one leaving. The amount of this shift may be controlled by adjusting the magnitude of the current in the vertical wire. This shift is limited only by the disappearance of two of the lobes ${ }^{3}$ forming two cardioid patterns at right angles, thus giving two equisignal zones displaced $180^{\circ}$ instead of four displaced at various angles.

It has previously been mentioned that in the original beacon system the goniometer primaries were set at an angle of $120^{\circ}$ to secure a narrow course. When means are employed to bend the course this is not advisable. There is danger of one lobe shrinking so far that it intersects the adjacent lobe on the wrong portion of its contour. This will be apparent from Figure 9. Four courses are present so far as the ear can detect, but as the lobe decreases the course will no

3 E. Z. Stowell, A Unidirectional Radiobeacon for Aircraft, B. S. Jour. of Research; December, 1928. 
longer shift properly. Furthermore, the course at this intersection is not sharp enough to be used. In order to prevent this in beacon

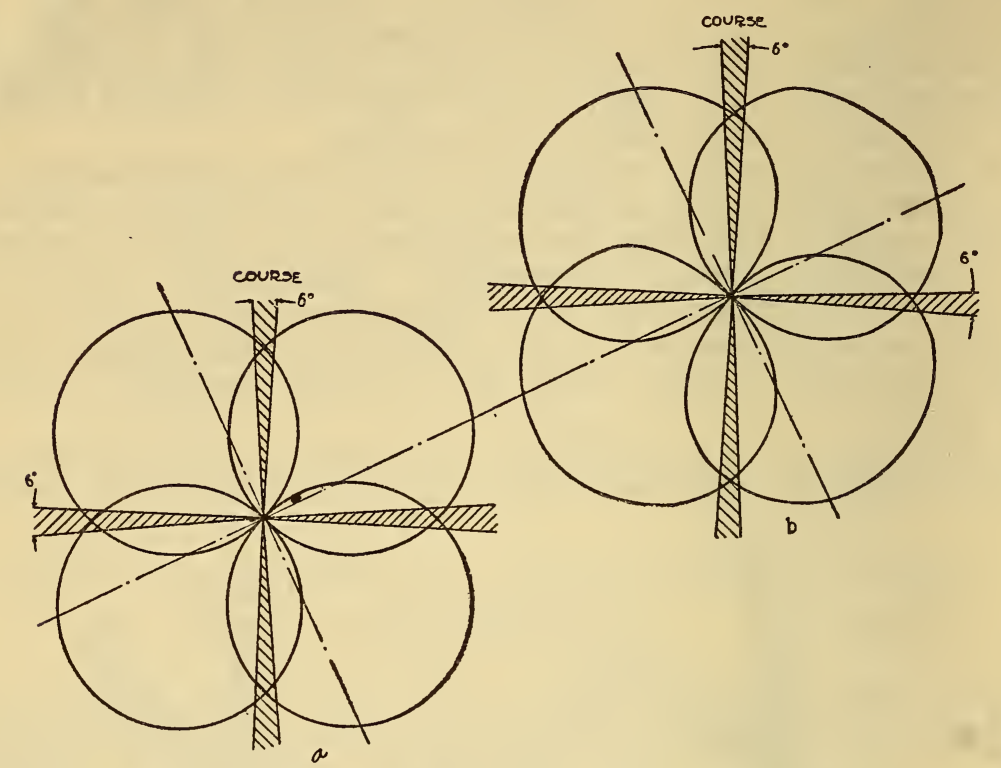

FIGURE 6.-Field intensity pattern of typical radio range. Goniometer primaries set at $90^{\circ}$

(a) Theoretical.

(b) Observed.

installations the primaries are frequently set at $90^{\circ}$ when the courses are to be bent.

\section{APPLICATION OF THEORY}

Having decided upon a method for aligning the courses of the radio range with the airways, the next problem was to develop a means of
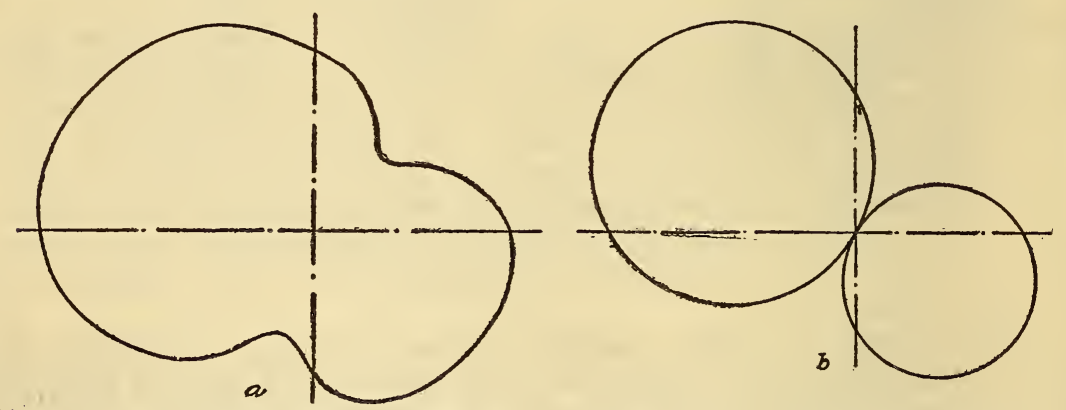

FrgURE 7.-Field intensity pattern of one phantom loop antenna and vertical antenna energized simultaneously

(a) Observed.

(b) Theoretical.

applying it to the beacon installations. The radio ranges at Hadley Field, New Brunswick, N. J., and Bellefonte, Pa., were selected for these tests. 
The airway route through Bellefonte has a $14^{\circ}$ bend at Bellefonte and, consequently, the antenna effect method was employed.

A vertical wire antenna was installed on the radio range tower and this was tuned to resonance by a suitable series inductance. The
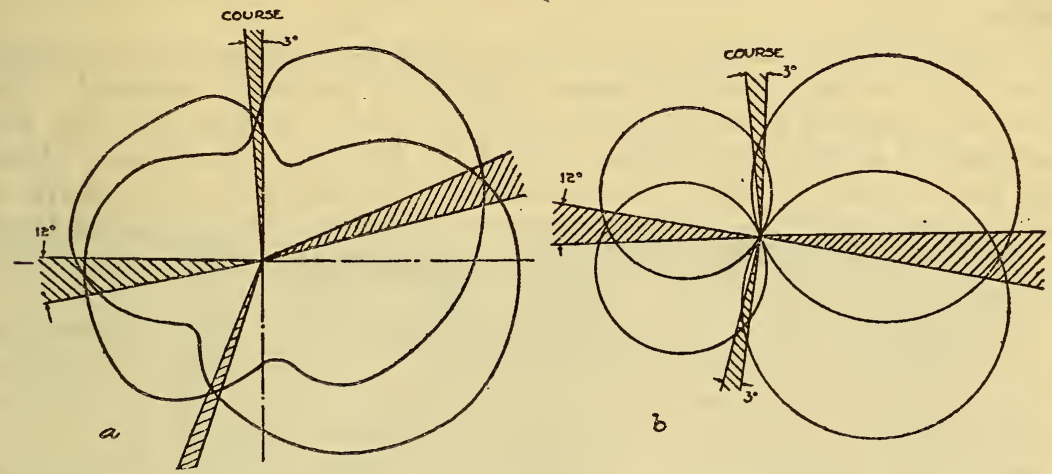

FIGURE 8.-Field intensity pattern of radio range employing a vertical antenna in addition to the loops. Goniometer primaries set at $120^{\circ}$

(a) Observed.

(b) Theoretical.

circuit was of sufficiently high resistance to have a broad resonance curve.

The antenna was at first excited by coupling it to the master oscillator. The results from this were poor, and analysis showed that a

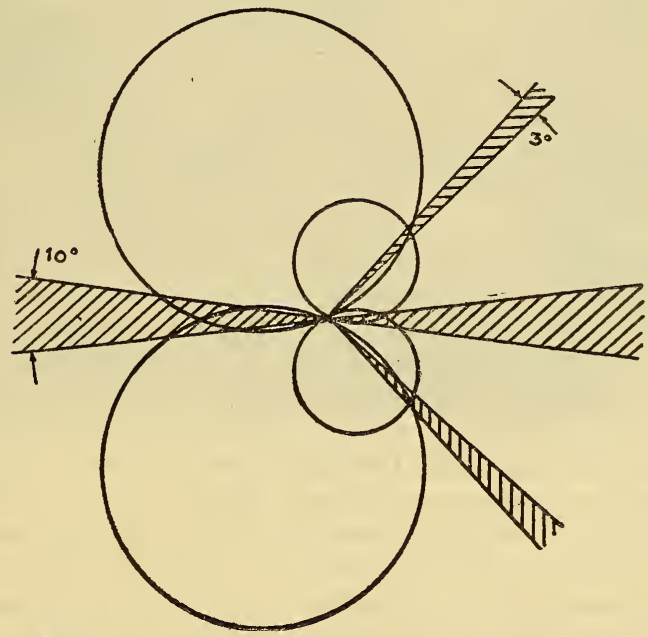

Figure 9.-Field pattern of Figure 8 with incorrect time phase of vertical antenna

pronounced phase shift occurred between the master oscillator and the goniometer, due to the particular coupling circuits employed. To eliminate this, a coupling coil was inserted in the common goniometer primary circuit, and the antenna coupled to this. This resulted in excellent control of the amount of shift, displacements of $28^{\circ}$ being 
readily obtainable. The goniometer primaries were set at $90^{\circ}$ for this work.

The final adjustment was made by controlling the degree of the antenna coupling. It is necessary that the antenna be in tune to prevent dephasing; consequently, detuning will not accomplish the desired result.

It was also necessary to have an accurate knowledge of the amount of shift in order that the course might be properly oriented. For first adjustments a receiving set using a 6 -foot vertical antenna was mounted in a truck and driven around the loops at a distance of about 100 yards from the base of the tower. Readings were very sharp at this distance and the $14^{\circ}$ bend was easily checked. By locating landmarks from compass bearings it was possible to align the course with the airways.

In order to secure the observed field intensity patterns shown the same receiving set was employed with its vertical pole antenna. The output was connected to a calibrated thermoelement and the signal intensity adjusted to keep the tubes under saturation at all times. In employing the vertical wire antenna for distorting the pattern the receiving set was stationary and the goniometer rotated through $360^{\circ}$. With the series resistance method, the set was moved around the beacon tower.

Discrepancies between observed and theoretical patterns are due chiefly to phase shift between the loop antenna currents and the vertical wire antenna current.

For a further check the truck was taken to Snowshoe Junction to the west and to Aaronsburg to the east. (These are, respectively, 10 and 20 miles from the radio range.) The readings here checked with those taken at the beacon tower to within half a degree, which is well within the accuracy of measurement. As a final check, the course was flown over, and again the readings checked those taken at the beacon. From this and from data secured on later occasions, it was decided that an accuracy of $1^{\circ}$ could be expected from measurements made at the beacon tower in spite of the predominating induction field.

Hadley Field, besides being the eastern terminus of the Transcontinental Airway, has two other air lines entering it. Hartford to the north, and Washington to the south, need to be served by the same radio range. Washington and Hartford lie in a straight line through Hadley Field, but the course to the west is not at $90^{\circ}$ to these. This presented an opportunity to use the first method described, namely, that of inserting resistance in the link circuit of one of the goniometer primaries, which, as before explained, shifts the $90^{\circ}$ courses.

With the current in one goniometer primary approximately one-half the value of the other, the courses were shifted the desired amount.

Here, also, it was found desirable to adjust the goniometer primaries to $90^{\circ}$ in order that the courses might be of equal power. This change in relative field intensity of the two phantom loops has another very advantageous effect in that it sharpens the course to a considerable degree. By referring to Figure 5 it will be seen that since the intersection of the smaller figure-of-eight comes at the point of rapid change of the large figure-of-eight, the resulting course is quite sharp. By measurement the courses have been found to be approximately 
B. S. Journal of Research, RP155

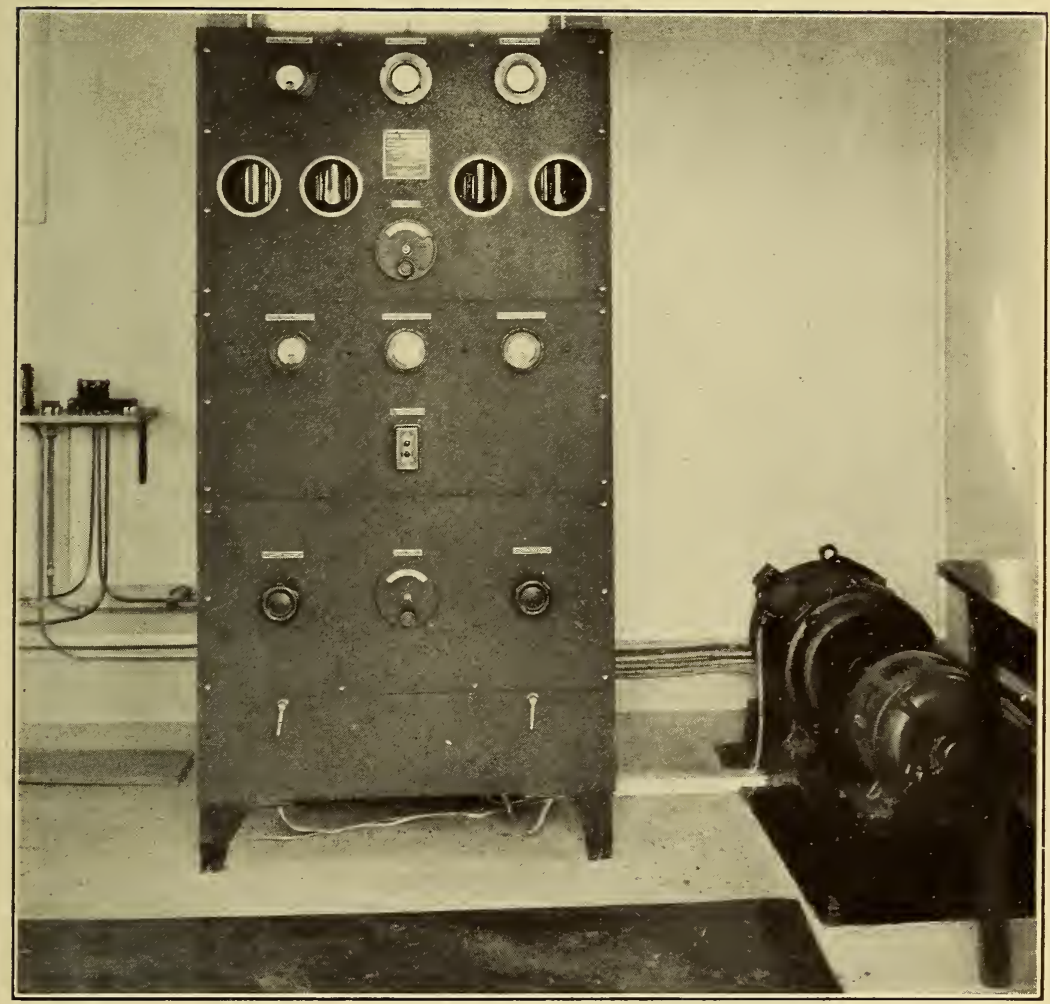

Frgdre 10.-Radio range installation at Hadley Field, N. J. 
$3^{\circ}$ wide. This is much sharper than the usual course resulting from a $90^{\circ}$ separation of the goniometer primaries.

The courses were checked here in a manner similar to that used at Bellefonte.

One other modification of the system is possible. When using a vertical wire antenna it may be keyed by a relay in the same circuit as the one goniometer primary. The result of this will be to affect only one phantom loop and thus shift both the $90^{\circ}$ and the $180^{\circ}$ courses.

With these three means at hand it becomes a relatively simple matter to satisfy almost any requirement where the number of courses to be served does not exceed three, and also a great number of 4-course air terminals. The adjustments are not so critical as to be unstable and once made, remain indefinitely. Very little extra apparatus is required and the maintenance is not difficult. All the requirements for the aural type of radio range, as listed in the early part of this paper, are therefore fulfilled.

Washington, August 8, 1929. 